\title{
A Map-based Lateral and Longitudinal DGPS/DR Bias Estimation Method for Autonomous Driving
}

\author{
S. S. Rathour, Ali Boyali, Lyu Zheming, Seiichi Mita, and Vijay John
}

\begin{abstract}
Autonomous driving requires continuous and reliable centimeter level positioning accuracy for acceptable lane level navigational performance. Centimeter level positioning accuracy cannot be achieved using a conventional DGPS/DR. To deal with the above problem this paper proposes the novel and effective bias/shift estimation of DGPS/DR. On this paper, a surveyed precise map is used that is consisting of waypoints with centimeter level positioning accuracy for the lateral and longitudinal directions. The time history of DGPS/DR waypoints are compared with the closest set of waypoints from surveyed precise map. Both a straight line fitting method and a sliding curve method have been used in order to match the shape of the DGPS/DR trajectory with surveyed precise map. For lateral and longitudinal DGPS/DR bias estimation, we have adopted a disturbance observer. Finally, experiments were conducted to prove the feasibility of the proposed algorithm for the shift estimation of DGPS/DR. This paper also compares the experimental results of GPS/DR with the ones using RTK GPS/DR during autonomous driving.
\end{abstract}

Index Terms-RTKt, DGPS bias, Map based DGPS bias estimation, DGPS based autonomous driving.

\section{INTRODUCTION}

In the recent time promising demonstrations of selfdriving car in real conditions have been carried out using market available sensors [1]. For most of the self-driving car demonstrations a custom made or informative digital map of the route to be followed by professional map making companies has been used. For implementing the reliable and continuous navigation capability, a vehicle satellite positioning system is augmented with other sensors such inertial measurement unit, odometer and exteroceptive sensors such as camera and LIDAR [2], [3]. Differential correction obtained by using GPS pseudo-range code (DGPS) can achieve an accuracy within $1-5 \mathrm{~m}$, on the other hand, the usage of GPS carrier phase information such as real time kinematics (RTK) GPS can achieve centimeter level positioning accuracy.

In case of loss of GPS signal due to environmental causes leads to the degradation of positioning accuracy [4], [5]. In such scenario the bias or the shift in the position should be estimated online and compensated for safety reasons.

Map matching algorithms have been used extensively in prior works to match the position from the positioning system to one on the road network in the map. Usually, the time history of the trajectory is matched with the shape of

Manuscript received July 6, 2017; revised August 20, 2017.

S. S. Rathour, Ali Boyali, Lyu Zheming, Seiichi Mita, Vijay John are with Research Center for Smart Vehicle, Toyota Technological Institute, Nagoya, Japan (e-mail: \{swarn,ali, smita, vijayjohn\}@ toyota-ti.ac.jp, lvzheming@gmail.com). the road network. Map matching technique generally include geometrical analysis such as point to point matching, point to arc matching and curve to curve matching, probability theory, extended Kalman filter, and hidden Markov model [6], [7]. In order to reduce localization error, [8]-[10] proposed the method that uses lane detection method to improve the GPS/DR error estimation. However, in case of no lane visual information such method is susceptible of noise addition. Various authors [4], [5] proposed the use of enhanced map-matching algorithm for urban environments, using topological information of digital map, as well as historical information of the route followed by a fuzzy rule for real time application.

Autonomous driving requires continuous and reliable centimeter level positioning accuracy for acceptable lane level navigational performance. Map matching algorithm augmented with exteroceptive sensors such as camera and LIDAR can be used to derive DGPS/DR lateral bias, but longitudinal bias is difficult to estimate [1]. In order to overcome the limitation of map matching algorithm, as well as dependency of lane information and visible feature for exteroceptive sensors for bias estimation we propose the use of surveyed precise map built by using RTK GPS with centimeter level positioning accuracy. The surveyed precise map consists of waypoints at $10 \mathrm{~cm}$ distance interval. In order to predict the DGPS/DR lateral and longitudinal bias we use the time history of position trajectory.

This paper has been divided into five sections. The first section briefly introduces the objective of the paper followed by the brief literature survey and a brief introduction of the paper. Second section defines the terminology used and, mathematical formulation of disturbance observer for bias estimation. Third section deals with the formulation of straight line fit algorithm and curve to curve matching. Fourth section explains the experimental results. Finally, last section is for conclusion.

\section{MAP-MATCHING ALGORITHM}

\section{A. Problem Definition}

Two coordinated system, earth fixed coordinate system $X Y$ and body fixed coordinated system $x y$ has been used (Fig. 1) for defining the variables and control application. For map matching three definitions for set of waypoints representing section of the map $C$ (Fig. 1), set of waypoints representing the vehicle trajectory $T$ and finally full map $M$ are defined as follows:

Test Segment: Test segment, $T=\left(T_{n 1}, n 1=1,2 \ldots n\right)$ is the time history of the position trajectory from DGPS/DR as shown in Fig. 1. 


$$
T_{n 1}=(x, y, \psi, \dot{\psi}, v, \dot{v})
$$

where $(x, y)$ is with respect to $X Y, \psi$ vehicle heading , $\dot{\psi}$, vehicle yaw rate, $(v, \dot{v})$ represent the vehicle velocity and acceleration respectively.

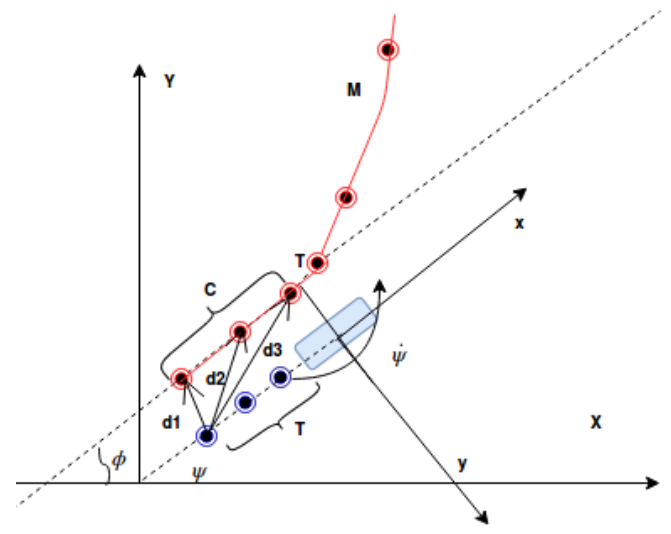

Fig. 1. Earth based coordinate system and body based coordinate system.

Candidate Segment: Candidate segment, $C=$ $\left(C_{n 2}, n 2=1,2 \ldots n 2\right)$ is the set of waypoints closest to test segment $T$.

$$
\begin{aligned}
& \mathrm{C}_{\mathrm{n} 2}=\left(x_{n 2}, \mathrm{y}_{n 2}, \phi\right) \\
& \phi=\operatorname{atan} 2\left(x_{n 2}, \mathrm{y}_{n 2}\right)
\end{aligned}
$$

where $\left(x_{n 2}, \mathrm{y}_{n 2}\right)$ is waypoint in digital map with respect to $X Y$. Dynamic time wrapping method is used to find $C$ corresponding to $T$ from $M$. $T_{1}=C_{1}$ i.e. $d_{1}=\min \left(d_{1}, d_{2}, d_{3}\right)$, where $d_{i}=\left(\operatorname{norm}\left(\mathrm{T}_{\mathrm{i}}-\right.\right.$ $\mathrm{C}_{\mathrm{i}}$ ) and $\psi-\phi \leq 90$.

Digital Map: A digital map, $M=\left(D_{n 3}, n 3=\right.$ $1,2 \ldots n 3)$ is the set of the position waypoints collected using RTK enabled GPS for autonomous driving.

$$
D_{n 3}=\left(x_{n 3}, \mathrm{y}_{n 3}, \kappa\right)
$$

where $\left(x_{n 3}, y_{n 3}\right)$ is waypoint in digital map with respect to $X Y, \kappa$ is path curvature at the corresponding waypoint.

\section{B. Disturbance Observer}

Disturbance observer is a useful tool to account for unmolded or undesired force acting on the system, widely used in the field of robotics and control application. In this study we use the principle of disturbance observer to estimate the DGPS/DR lateral and longitudinal bias, as the average or mean of point to point bias between $C$ and $T$ is not always good enough for compensating the DGPS/DR bias. For the disturbance observer formulation, we use state space model representation, as in (4).

$$
\begin{gathered}
x(k+1)=A x(k)+B(u(k)+d(k)) \\
d(k+1)=d(k) \\
A=\left[\begin{array}{ll}
0 & 0 \\
0 & 0
\end{array}\right], B=\left[\begin{array}{ll}
1 & 0 \\
0 & 1
\end{array}\right], L=\left[\begin{array}{ll}
L & 0 \\
0 & L
\end{array}\right]
\end{gathered}
$$

In (4) $x(k+1)$ represents the absolute position derived from the DGPS/DR positioning system, $u(k)$ represents the closest point corresponding to $x(k+1)$ in the digital map and $d(k)$ represents the DGPS/DR lateral and longitudinal bias. Equation 5 represents the real system dynamics of the DGPS/DR bias.

$$
\begin{gathered}
\vec{d}(k+1)=\vec{d}(k)+L *(d(k)-\vec{d}(k)) \\
e(k+1)=e(k)(1-L B)
\end{gathered}
$$

Equation 7 represents the numerical estimation of (5), choosing appropriate $L$ (observer gain) can give good estimate of real bias. Subtracting (5) and (7) gives the error equation of estimated error $e(k+1)$ and real bias error $e(k)(1-L B)(8)$. As $B$ is identity matrix, $L$ should lies between $0-1$ to reduce $e(k+1)$ to zero.

Substituting $d(k)$ from (1) in (7) will give (9), rearranging (9) gives (10).

$$
\vec{d}(k+1)=d(k)+L B(x(k+1)-u(k)-\vec{d}(k))
$$

$$
\vec{d}(k+1)=(1-L B) d(k)+L B(x(k+1))-L B u(k)
$$

\section{Section Matching Algorithm}

For matching the test segment with the digital map, two approaches are followed:

\section{A. Straight Line Fit}

In the case of straight line test segment $T$ as shown in Fig. 2 (bottom left corner) by dotted line and corresponding candidate segment $C$ by dashed line, linear straight line equation is used to fit the $T$ and $C$. Hence, two linear straight line equation can be derived one defining $T$ i.e. (11) and other defining $C$ (12). The advantage of using straight line fit for lateral and longitudinal DGPS/DR bias is that any jump or outlier in the time history of DGPS/DR can be easily avoided. Straight line fit to the $T$ and $C$ can easily give both longitudinal as well as lateral bias. In case of map segment aligning perfectly with north axis or east axis straight line fit cannot give lateral and longitudinal DGPS/DR bias at the same time. In such condition two measurements are necessary to compute the DGPS/DR bias separately.

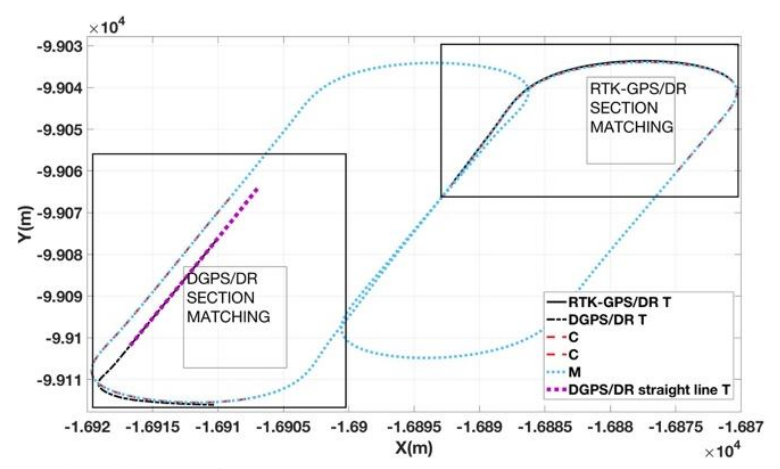

Fig. 2 Time history of DGPS/DR and RTK-GPS/DR test segment $T$ (solid and dotted line), the corresponding candidate segment (dashed red lien) $C$ and full digital map with blue solid line

$$
\begin{aligned}
& C(y)=a * C(x)+B \\
& T(y)=a * T(x)+B^{\prime}
\end{aligned}
$$

In order to match $T$ with $C$ lateral and longitudinal bias 
can be derived easily using (11) and (12). $C(y), T(y)$ and $C(x), T(x)$ represent the RTK-GPS and DGPS latitude and longitude position respectively, $a$ represents the slope of the straight line and $B, B^{\prime}$ finally represents the y-intercept. Adding the derived bias to $T$ gives exactly $C$.

\section{B. Curve to Curve Matching}

To overcome the limitation of straight line segment based lateral and longitudinal bias computation, curved section matching approach was used. The proposed method is similar to one used in map to map matching algorithm [11] i.e. dynamic time wrapping algorithm. Fig. 3 shows the plot of $\mathrm{T}$ and $\mathrm{C}$ (bottom left zoomed portion of Fig. 1).

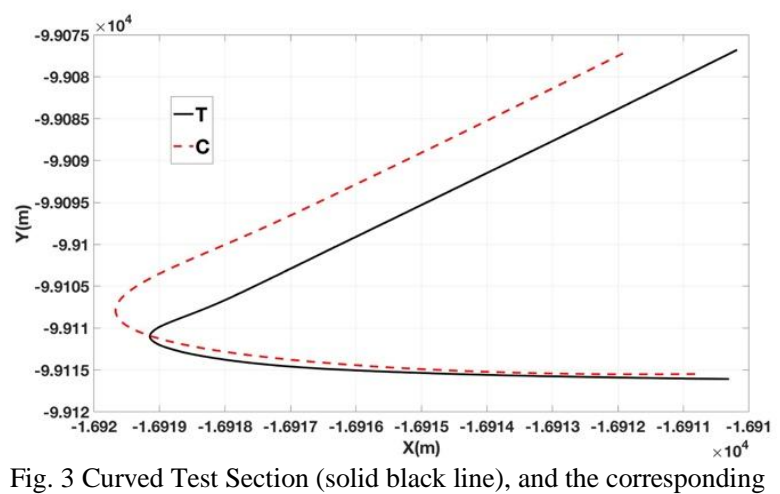
candidate segment (dashed red line).

Point to point matching is used to identify the candidate node on $M$, given a candidate node set of waypoints originating from the candidate node are used to define $C$ [7].

$$
\begin{gathered}
\operatorname{norm}\left(C_{i+1}-C_{i}\right)=\operatorname{norm}\left(T_{i+1}-T_{i}\right) \\
d_{i}=\sum_{i=1}^{l 1} \sum_{j=1}^{l 2} \operatorname{norm}\left(C_{i+j-1}(x, y)-T_{j}(x, y)\right)
\end{gathered}
$$

Once the possible candidate segment waypoint has been found, the length of $C$ i.e. $l 1$ is chosen in such a way that it should enclose the test section $T$ (i.e. $l 1>l 2$ ). For one to one-point matching in $T$ and $C$, it is made sure that distance between consecutive waypoint of $\mathrm{T}$ should be same as that of $C$ (13). $T$ is slided over the $C$ and sum of the total Euclidian distance calculated using (14) is stored in $d_{i}$. Finally the index of the minmume $\left(d_{i}\right)$ i.e. $\left(d_{i}=\right.$ min $\left.\left(d_{i}\right)\right)$ gives the starting index of the $C$ segment matching closely with $T$ i.e. $T m$ (15). As mentioned above both $T$ and $T m$ have equal number of sampled position points the lateral and longitudinal error can be written as simple average $e_{i}$ (16).

$$
\begin{gathered}
\text { Tm }=C_{i+\text { imin }}, i=i+i m i n: i+i m i n+l 2 \\
e_{i}=\frac{\sum_{j=1}^{l 2}\left(C_{i m i n}+j-1(x, y)-T_{j}(x, y)\right)}{l 2}
\end{gathered}
$$

\section{DGPS/DR BIAs EsTIMATION EXPERIMENTAL RESULT}

To prove the DGPS/DR bias estimation methods as proposed in section-III, data collection was done using the test vehicle (Toyota Estima Hybrid) within the campus of Toyota Technological Institute, Nagoya Japan. The test vehicle is equipped with a satellite based positioning system
(GPS) augmented with dead reckoning sensor system provided by APPLANIX 610(A Trimble Company). The GPS is capable of using GPS carrier phase information (GPS-RTK) for differential position correction. The positioning system GPS/DR has the option to choose between GPS differential correction (i.e. GPS pseudo range code or GPS carrier phase information). All the experiments such as data collection for map building or autonomous driving GPS/DR sensor system operates at $200 \mathrm{~Hz}$ frequency. To begin with, position data to build the map was collected by manual driving. The collected position data was processed offline to remove any jumps in GPS/DR position, steady state position data (i.e. when the test vehicle was idle or not moving). After smoothing the position data was resampled and interpolated to make sure that $\operatorname{norm}\left(D_{i+1}-\right.$ $\left.D_{i}\right)=10 \mathrm{~cm}$. All the software package for recording as well as autonomous control was written in ROS (Robot operating system). Three set of experiment were performed to prove the efficiency of proposed algorithm.

\section{GPS-RTK/DR Based Autonomous Driving}

For the autonomous driving only steering control was performed, the speed of the test vehicle was controlled manually by the person seating behind the wheels. Firstly, autonomous driving experiment using RTK-GPS/DR was carried out. The digital map $D$ was used for autonomous driving (Fig. 2).

After the experiment time history of RTK-GPS/DR controlled trajectory was processed offline in order to validated the proposed map based positioning system bias estimation algorithm. The curved section $T$, shown in Fig. 2 (top right corner, solid black line) was selected for bias estimation. As per geometrical matching the closest candidate segment with respect to $T$ on $D$ was computed $(C)$. The test segment $T$ was slided over $C$ to find the best match (14). Finally the indices of minimum $d_{i}$ (i.e. $i m i n$ ) give the starting indices of matched segment $T m$. Fig. 4 shows the plot of $T+d, T+e$ and $T m$.

The main objective of this experiment was to show the lateral and longitudinal error range during RTK-GPS/DR based autonomous driving. Due to some inaccuracy of the controller and path smoothing the test vehicle cannot follow the curve section accurately, but still follows the defined path $D$ within an error range of $\pm 30 \mathrm{~cm}$ which is acceptable for lane level navigational performance.

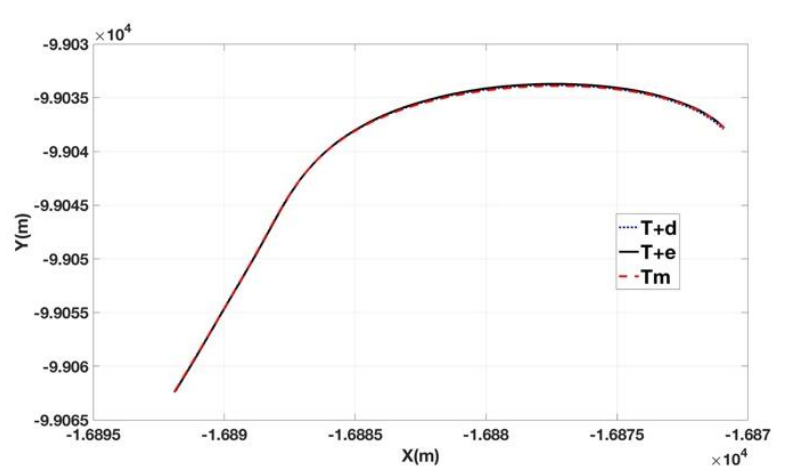

Fig. 4. Plot of $T_{m}$ (Red dash and dot line), $T+d$ (dotted purple line) and ), $T+e$ (solid black line).

Fig. 5 shows the xy-plot of point to point lateral and longitudinal error between the $T_{m}$ and $T+d$ (dashed orange 
line), $T_{m}$ and $T+e$ using average error (solid blue line). From Fig. 4 it can be concluded that lateral and longitudinal error between $T_{m}$ and $T+d$ based on disturbance observer lies within $\pm 10 \mathrm{~cm}$ which is accepted for autonomous driving.

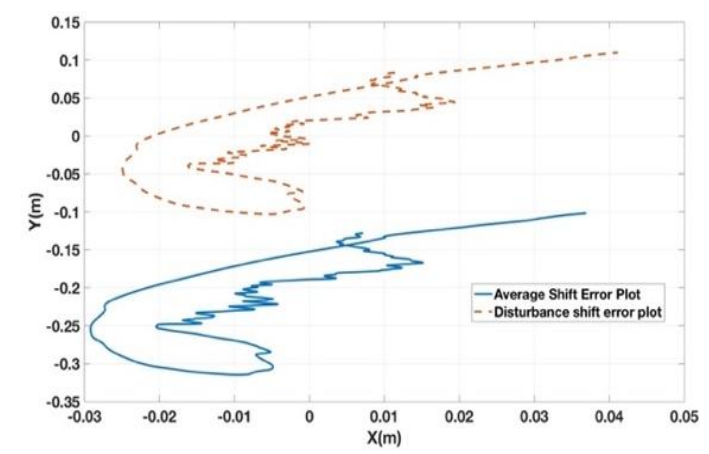

Fig. 5. XY plot of lateral and longitudinal error derived using point to point difference between the $T_{m}$ and shifted $T$ using average error (solid blue line), and disturbance observer error (dashed orange line).

\section{Straight Line Fit}

For the next experiment, position data was collected using DGPS/DR mode in manual driving mode. During this experiment GPS carrier phase information was not used only GPS pseudo-range information was used. The main objective of the experiment is to find the lateral and longitudinal DGPS/DR bias, compensating which can make the test vehicle follow the map accurately within the defined error limit. The straight line section shown in bottom left corner of Fig. 2 was selected as test segment $T$. Geometrical approach to find the closest point to define the candidate Section $C$ (Fig. 2) was used. The straight line fit as explained in Section III. B was used to estimate lateral and longitudinal shift.

Fig. 6 shows the plot of matched section $T_{m}$ (red dashed line), shifted test section $T+e$ using straight line fit (dotted red line) and finally shifted $T+d$ using the disturbance observer (dashed blue line). In straight line section straight line fit perform better than disturbance observer.

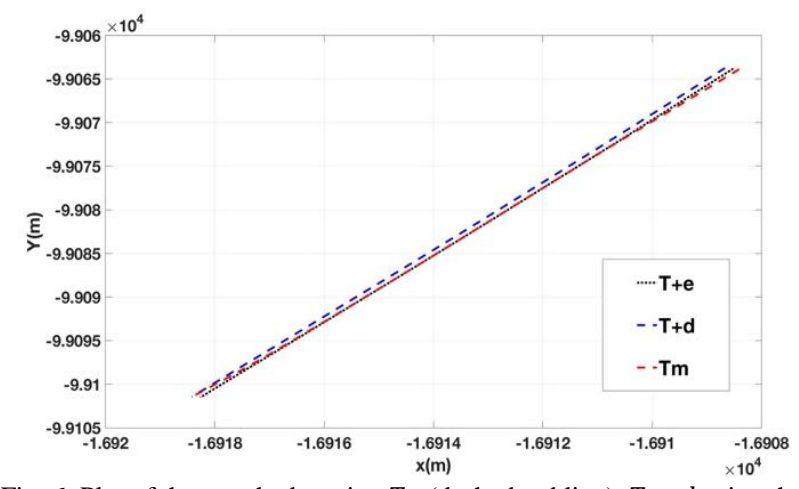

Fig. 6. Plot of the matched section $T_{m}$ (dashed red line), $T+d$ using the bias derived from disturbance observer (dashed blue line) and $T+e$ (red dotted line).

\section{E. Curve Section Fit}

Similar approach as followed in straight line fit to find the candidate segment $C$ (i.e. geometrical matching) was followed for curve section also. $T$ and $C$ for this experiment has been shown in Fig. 2.

For curved section straight line fit cannot be used to compute lateral and longitudinal bias estimate. Hence, curved to curve matching algorithm as explained in sectionIII was used to derive the longitudinal and lateral DGPS/DR bias. Test segment $T$ was slided (14) over the candidate segment $C$ to find the best matching segment. Finally, lateral and longitudinal error was derived using (16) and (4-10).

Fig. 8 shows the plot of the lateral and longitudinal error between the matched segment $T_{m}$ and test segment $T$ (solid black line). From the error plot it can be concluded that lateral and longitudinal bias between $T_{m}$ and $T$ lies within $60 \mathrm{~cm}$ to $100 \mathrm{~cm}$. Fig. 7 also shows the lateral and longitudinal error between the $T_{m}$ and $T+e$ using the average error based on (16) by dotted purple line and $T_{m}$ and $T+d$ (disturbance observer (4-10)) by dashed red line.

From the Fig. 7 it can be concluded that estimation of lateral and longitudinal error based on disturbance observer does a better job than simple average lateral and longitudinal bias estimation.

\section{F. Autonomous Driving using GPS-RTK/DR and Compensated DGPS/DR}

Finally, autonomous driving experiment were conducted to prove the feasibility of the proposed algorithm in order to compute lateral and longitudinal bias. The map shown in Fig. 2 was used for the experiment. At first GPS carrier phase information was used (GPS-RTK/DR) i.e. position accuracy was within $1-10 \mathrm{~cm}$.

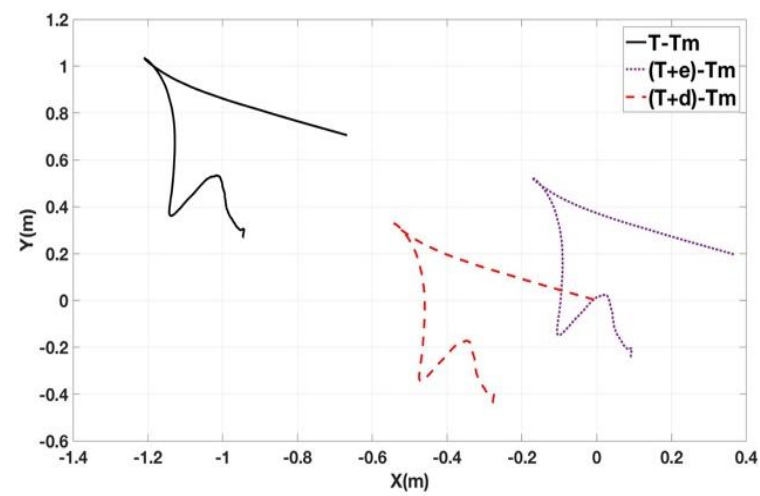

Fig. 7. XY plot of lateral and longitudinal error derived using point to point difference between the $T_{m}$ and $T$ (solid black line), point to point difference between the $T_{m}$ and $T+e$ (dotted purple line), and $T_{m}$ and $T+d$ (dashed red line).

In case of DGPS/DR based autonomous driving, GPS carrier phase information was not used. For DGPS/DR based autonomous driving, at first the lateral and longitudinal bias is computed by manually following the map waypoint. The derived lateral and longitudinal bias by manual driving is used to compensate the positioning system during autonomous driving using DGPS/DR. Fig. 8 \& Fig. 9 shows the time history of lateral and heading error for autonomous driving using GPS-RTK/DR (dashed blue line) and compensated DGPS/DR (solid orange line). From Fig. 8 and Fig. 9 it can be concluded that error limit lies within the acceptable range and compensated DGPS/DR is capable of following the map waypoints within the desired error limit. The error plot in Fig. 8 and Fig. 9 does not superimpose over each other, because for autonomous driving only steering control is performed whereas speed is controlled manually. 


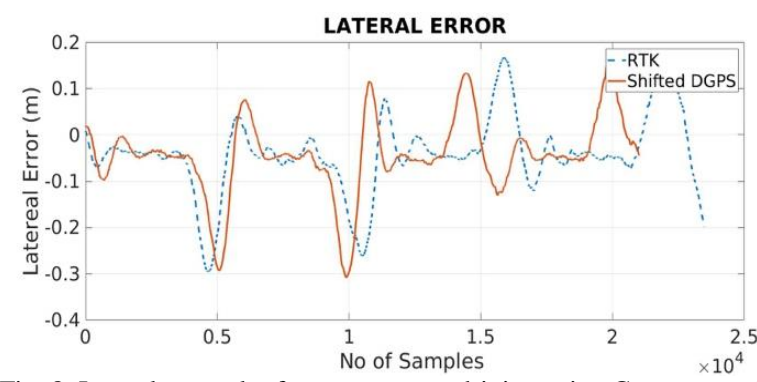

Fig. 8. Lateral error plot for autonomous driving using Compensated DGPS/DR (solid orange line), GPS-RTK/DR (dashed blue line).

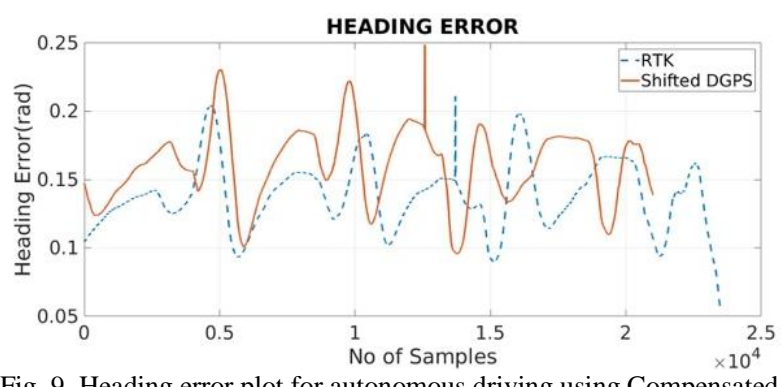

Fig. 9. Heading error plot for autonomous driving using Compensated DGPS/DR (solid orange line), GPS-RTK/DR (dashed blue line).

\section{CONCLUSINON}

The paper proposes the use of straight line fit for the lateral and longitudinal bias of DGPS/DR positioning system. The straight line fit algorithm does not depend on the stop line information of the enhanced digital map. The paper also proposes the use of disturbance observer for the estimation of lateral and longitudinal error in case of curved section. The proposed disturbance observer performs better than the simple average error estimation. The autonomous driving experiment using compensated DGPS/DR showed similar performance as (GPS-RTK/DR).

\section{REFERENCES}

[1] P. Furgale et al., "Towardautomateddrivingincitiesusingclose-tomarket sensors: An overview of the v-charge project," in Proc. IEEE Intell. Veh. Symp. (IV), Jun. 2013, pp. 809-816.

[2] D. B. 'Taille and R. Toledo-Moreo, "Creating enhanced maps for lanelevel vehicle navigation," IEEE Trans. Intell. Transp. Syst., vol. 11, no. 4, pp. 786-798, Dec. 2010.

[3] I. Miller, M. Campbell, and D. Huttenlocher, "Map-aidedlocalizationin sparse global positioning system environments using vision and particle filtering," J. Field Robot., vol. 28, no. 5, pp. 619-643, 2011.

[4] Z. Tao and P. Bonnifait, "Road invariant extended kalman filter for an enhanced estimation of GPS errors using lane markings," in Proc. IEEE/RSJ Int. Conf. Intell. Robots Syst., Hamburg, Germany, Sep. 2015, pp. 3119-3124.

[5] Z. Tao and P. Bonnifait, "Sequential data fusion of GNSS pseudoranges and dopplers with map-based vision system," IEEE Transactions on Intelligent Vehicles, vol. 1, no. 3, pp. 254-265, September-2016.

[6] C. Y. Goh, J. Dauwels, N. Mitrovic, M. T. Asif, A. Oran, and P. Jaillet, "Online map-matching based on Hidden Markov model for real-time traffic sensing applications," in Proc. 15th International IEEE Conference on Intelligent Transportation Systems, 2012.

[7] G. R. Jagadeesh, T. Srikanthan, and X. D. Zhang, "Online mapmatching based on Hidden Markov model for real-time traffic sensing applications," Journal of Navigation, vol. 57, pp. 429-440, 2004.

[8] B. H. Lee, J. H. Song, J. H. Im, S. H. Im, M. B. Heo, and G. I. Jee, "GPS/DR error estimation for autonomous vehicle localization," Sensors, vol. 15, no. 8, 2015.

[9] C. Wenk et al., "Addressing the need for map- matching speed: localizing global curve-matching algorithms," in Proc. 18th

International Conference on Scientific and Statistical Database Management, 2006, pp. 379-388.

[10]Z. Tao, P. Bonnifait, V. Fre' mont, and J. Ibañ ez-Guzman, "Mapping and localization using GPS, lane markings and proprioceptive sensors," in Proc. 2013 IEEE/RSJ Int. Conf. Intell. Robots Syst., Nov. 2013, pp. 406-441.

[11]M. Quddus, W. Ochieng, and R. Noland, "Current map-matching algorithms for transport applications: State-of-the art and future research directions," Transportation Research Part C: Emerging Technologies, vol. 15, no. 5, pp. 312-328, 2007.

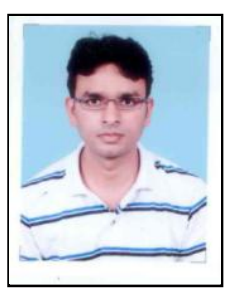

vehicles.

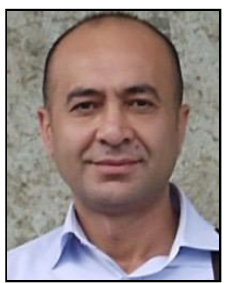

Ali Boyali received his bachelor degree from the Mechanical Engineering Faculty of the Istanbul Technical University in 1998, M.Sc. degree in automotive engineering in 2002, and his Ph.D. in automotive mechatronics in 2008 . He is now with the National Institute of Advanced Science and Technology in Japan as a post-doctoral research fellow. His recent researches focus on the development robotic wheelchairs that can be commanded by the recognized gesture and postures of the people with mobility impairment.

Lyu Zheming is first year master student at Toyota Technological Institute, Nagoya Japan. His current research interests include computer vision, machine learning with applications in autonomous vehicles.

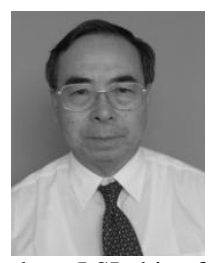

Seiichi Mita received the B.S. degree, the M.S. degree and the Ph.D. degree in electrical engineering from Kyoto University in 1969, 1971, 1989 respectively. He studied at Hitachi Central Research Laboratory, Kokubunji, Japan from 1971 to 1991 on signal processing and coding methods. He moved to Data Storage \$I\&\$ Retrieval Systems Division, Hitachi, Ltd. in 1991. He developed channel coding methods and these LSI chips for magnetic disk drives. Now, he is a professor of Toyota Technological Institute (TTI) in Nagoya since 1999 and a director of Research Center for Smart Vehicles at TTI. Currently, he is greatly interested in the research area of autonomous vehicles and sensing systems. $\mathrm{He}$ is a member of the Institute of Electronics, Information and Communication Engineers and the Institute of Image Information and Television Engineers in Japan. He is also a member of IEEE. He received the best paper award of IEEE Consumer Electronics Society in 1986 and the best paper and author awards of the Institute of Television Engineers in Japan in 1987 and 1992 respectively.

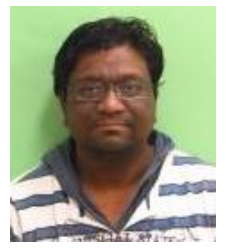

Vijay John received the M. S degree in information technology and robotics from Carnegie Mellon University, USA in 2005. He received the $\mathrm{Ph} . \mathrm{D}$ degree in computer vision from the University of Dundee, Dundee, UK in 2007. He then joined the University of Amsterdam in 2011 as a postdoctoral researcher, and was a visiting researcher at Philips Research, Netherlands during the summer of 2012. In 2014, he joined the Toyota Technological Institute, Nagoya, Japan, as a postdoctoral research fellow. Since 2017, he is an assistant professor at the Toyota Technological Institute. His current research interests include computer vision, machine learning with applications in autonomous vehicles and human motion analysis. 